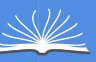

Global journals Inc.

(8)

\title{
Rare Case of Young Patient with Intraventricular Angiomatous Meningioma
}

By Gabriel Carvalho Heemann, Vinicius Rosa de Castro, Normando Guedes Pereira Neto, Camila Bocchi, Otavio Garcia Martins, Rafael Silva Paglioli \& Ricardo Chmelnistky Wainberg

Hospital São Lucas da Pontifícia Universidade Católica do Rio Grande do Sul Abstract- Pediatric meningiomas are rare and account for only 2.2\% of CNS tumors. In this age group, they are more frequently located in atypical sites, such as, mainly, the ventricular system, with a frequency of 8.8 to $13.6 \%$. Adding this to the fact that the angiomatous subtype constitutes only $2.1 \%$ of all meningiomas, the rarity of the case reported here i s corroborated. We report a 17-year-old female patient diagnosed with intraventricular angiomatous meningioma; she underwent surgical resection of the tumor in the body and frontal horn of the right lateral ventricle, and there was no neurological sequela. With a follow-up of 4 years, there was no recurrence and the patient had clinical stability. Intraventricular tumors usually have slow growth and reach considerable size until they cause symptoms and then are diagnosed. In addition, the tumor's deep location and proximity to eloquent areas make such tumors an neurosurgical challenge. The angiomatous subtype, due to the presence of hyper vascularization (consisting of more than $50 \%$ of vascular components), may, in some cases, hinder surgical resection as well as be erroneously diagnosed. However, surgical treatment aimed at total resection of the lesion remains the conduct of choice in the case reported here, especially in patients in the first two decades of life, in which the use of radiation is avoided. Specifically when it comes to the surgery, we chose a transcallosal approach that allows a good transoperative visualization of the lesion when located in the body and frontal horn of the lateral ventricle.

GJMR-A Classification: NLMC Code: WL 140

Strictly as per the compliance and regulations of:

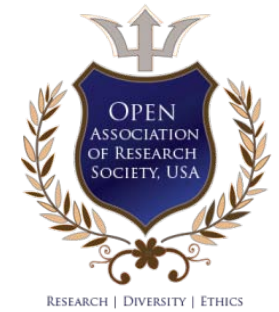

(C) 2021. Gabriel Carvalho Heemann, Vinicius Rosa de Castro, Normando Guedes Pereira Neto, Camila Bocchi, Otavio Garcia Martins, Rafael Silva Paglioli \& Ricardo Chmelnistky Wainberg. This is a research/review paper, distributed under the terms of the Creative Commons Attribution-Noncommercial 3.0 Unported License http://creativecommons.org/licenses/by-nc/3.0/), permitting all non-commercial use, distribution, and reproduction in any medium, provided the original work is properly cited. 


\title{
Rare Case of Young Patient with Intraventricular Angiomatous Meningioma
}

\author{
Gabriel Carvalho Heemann ${ }^{\alpha}$, Vinicius Rosa de Castro ${ }^{\circ}$, Normando Guedes Pereira Neto ${ }^{\circ}$, \\ Camila Bocchi ${ }^{\omega}$, Otavio Garcia Martins ${ }^{*}$, Rafael Silva Paglioli ${ }^{\S} \&$ Ricardo Chmelnistky Wainberg ${ }^{x}$
}

Abstract- Pediatric meningiomas are rare and account for only $2.2 \%$ of CNS tumors. In this age group, they are more frequently located in atypical sites, such as, mainly, the ventricular system, with a frequency of 8.8 to $13.6 \%$. Adding this to the fact that the angiomatous subtype constitutes only $2.1 \%$ of all meningiomas, the rarity of the case reported here $i$ s corroborated. We report a 17-year-old female patient diagnosed with intraventricular angiomatous meningioma; she underwent surgical resection of the tumor in the body and frontal horn of the right lateral ventricle, and there was no neurological sequela. With a follow-up of 4 years, there was no recurrence and the patient had clinical stability. Intraventricular tumors usually have slow growth and reach considerable size until they cause symptoms and then are diagnosed. In addition, the tumor's deep location and proximity to eloquent areas make such tumors an neurosurgical challenge. The angiomatous subtype, due to the presence of hyper vascularization (consisting of more than $50 \%$ of vascular components), may, in some cases, hinder surgical resection as well as be erroneously diagnosed. However, surgical treatment aimed at total resection of the lesion remains the conduct of choice in the case reported here, especially in patients in the first two decades of life, in which the use of radiation is avoided. Specifically when it comes to the surgery, we chose a transcallosal approach that allows a good transoperative visualization of the lesion when located in the body and frontal horn of the lateral ventricle.

\section{InTRODUCTION}

$\mathrm{M}$ eningiomas have a progressively higher incidence with increasing age, with a mean age of presentation of 65 years. They are, therefore, the most frequently reported tumors of the central nervous system (CNS) in adulthood. ${ }^{1}$ However, cases in children and adolescents are rare, representing 2.2 to $2.6 \%$ of CNS tumors. ${ }^{1,2}$ In such age group, are more often located in unusual sites, such as in the ventricular system. ${ }^{3}$

Such intraventricular meningiomas have the particularity of being slow growing and reaching considerable size until they become symptomatic. ${ }^{4}$ In addition, the deep location and relationship with underlying eloquent areas make tumor resection a neurosurgical challenge. ${ }^{5,6}$ In view of this, and that the angiomatous subtype- defined as presenting more than $50 \%$ of vascular components on microscopic analysis

Author $\alpha \sigma \rho \omega ¥ \S \chi$ : Department of Neurosurgery, Hospital São Lucas da Pontifícia Universidade Católica do Rio Grande do Sul, Brasil. e-mail: gabriel.heemann@gmail.com analysis - constitutes only $2.1 \%$ of all meningiomas, rarity is credited to the case reported here. ${ }^{7}$ We emphasize that this is possibly the first report of a patient in the first two decades of life with angiomatous meningioma in an intraventricular site.

In this report, we aim to expose our neurosurgical experience in a case with rare variants and perform a literature review on the main aspects that we deem necessary to support our approach.

\section{CAse Report}

A 17-year-old female patient, previously healthy, presented retro-orbital headache for 3 months, followed by blurred vision and double vision. Physical examination revealed just a convergent strabismus due to paresis of the right lateral rectus muscle. The cranial magnetic resonance (MRI) showed an expansive lesion in the frontal horn of the right ventricle, just in front of the foramen of Monro, with dimensions of $2.0 \times 1.3 \times 1.8 \mathrm{~cm}$ (AP $x L \times h)$ in the larger diameter cuts.

The lesion presented moderate hyperintensity with small hypointense foci on T2-weighted images. On $\mathrm{T1}$, it was isointense, and after contrast, it showed intense and homogeneous uptake, except in the same hypointense foci on T2. There were no signs of dilation of the supratentorial ventricular system (Fig. 1 A - D). Such radiological characteristics suggested the diagnosis of intraventricular meningioma, with areas of calcification. It was considered an occasional finding, since the topography was not compatible with the presenting symptoms.

The surgical treatment was performed in the same hospitalization, due to the risk of acute hydrocephalus. The approach was made via the transcallosal route, following the steps: dorsal decubitus and head in a neutral position; bicoronal incision; right frontal paramedian craniotomy, with lateral extension of $5.5 \mathrm{~cm}$ from the midline and $5.5 \mathrm{~cm}$ from the coronal suture forward; opening the dura mater in a "C" shape, with the base facing the midline; under microscopy, dissection of the inter-hemispheric fissure and retraction of the frontal lobe with placement of the fixed spatula (on a Leyla support) on the medial surface; identification of the cingulate gyrus and pericalosal arteries; callosotomy with $1,2 \mathrm{~cm}$ starting from the transition between the knee and the corpus callosum; identified a grayish vegetating lesion inside the right frontal horn, 
which had a soft consistency and were vascularized; resection of the lesion through coagulation and aspiration and by fragments, leaving a small residue adhered to the ependyma of the caudothalamic groove. Postoperatively uneventful and absence of new deficits, with diplopia and strabismus remaining.

$$
\text { Histopathological analysis and }
$$

immunohistochemical painel showed extensive vascularization and low mitotic index (Ki67 less than 2\%). In addition, that results were visualized: epithelial membrane antigen (EMA), positive; cytokeratin (CK), negative; progesterone receptor, negative; and glial fibrillary acidic protein (GFAP), positive (Fig.2 A-B). Thus, the diagnosis of angiomatous meningioma (WHO grade I) was confirmed. Postoperative MRI showed a small residual focus near the thalamostriate groove (Fig. 3 A-D and Fig. 4 A-D). After 6 months, the patient underwent strabismus correction with an ophthalmologist at another institution. Currently, with approximately 4 years of follow-up, she is asymptomatic and without evidence of recurrence of the residual lesion.

\section{Discussion}

Meningiomas are tumors that predominate in the fifth and sixth decades of life, with a mean age of presentation of 65 years. ${ }^{1}$ In general, they represent $36.4 \%$ of primary CNS tumors and approximately 24 $30 \%$ in adults. ${ }^{1,8}$ Nevertheless, in the pediatric population, the prevalence among CNS tumors varies between 0.4 and $4.6 \% .2^{3,9-13}$ The equivalence between genders also contrasts with what occurs in the adult population, which has a female to male ratio of $2: 1{ }^{1,2,14}$ This difference is believed to be due, mainly in the prepubertal period, to the absence of the effect of sex hormones on corticosteroid receptors in meningioma cells. ${ }^{10,15-17}$

In the first two decades of life, there is a higher incidence of grade II (atypical) and grade III (anaplastic) meningiomas, according to the WHO: 9.9 and 8.9\%, respectively. ${ }^{2,18}$ They are genetically and phenotypically more aggressive with a high frequency of brain invasion., ${ }^{9,14}$ Among the most frequent grade । meningiomas, the angiomatous subtype occurs in $2.8 \%$ of cases, ${ }^{2}$ and in $2.1 \%$ of all meningiomas at any age. ${ }^{19}$ Such subtype is defined when the vascular component exceeds $50 \%$ of the total area of the tumor. ${ }^{7,19}$ However, the differential diagnosis with hemangioblastoma and hemangiopericytoma is necessary, with immunohistochemistry and morphology having essential roles in the diagnostic confirmation: low MIB-1/Ki67 index and positivity for progesterone receptor, EMA, vimetin, cytokeratin, and desmoplaquin. ${ }^{7,19-23}$

The characteristics of angiomatous meningiomas, due to their rarity, are covered in few studies. ${ }^{7,19,24,25}$ They may present moderate to severe cerebral edema with a frequency of 74 to $88.9 \%{ }^{7,19}$ due to hypervascularization, increased blood pressure. capillary permeability and secretion of VEGF (vascular endothelial growth factor). ${ }^{19}$ On magnetic resonance imaging, they may show more signs of flow voids, rarely present necrosis and tend to have homogeneous enhancement to paramagnetic contrast. ${ }^{19,23}$

Meningiomas in pediatric patients present in atypical sites more frequently than in adults: lateral ventricles, skull base, posterior fossa. 2,3,10,15,26 Intraventricular location occurs in $11 \%$, compared to $0.3-$ $3 \%$ in all ages and $0.5-4.5 \%$ in adults. $2,10,27$

Intraventricular meningiomas (IVM) are located in $76 \%$ of cases in the lateral ventricles (most common on the left side); $16 \%$ in the third ventricle; and $7 \%$ in the fourth ventricle. ${ }^{5,27}$ There are studies suggesting that the lateral ventricles are the preferred site for pediatric IVMs. ${ }^{26,28}$ These originate from the choroid plexus, growing i $\mathrm{n}$ the $\mathrm{t} \mathrm{e} / \mathrm{a}$ choroidea. ${ }^{4}$ The vascularization of the tumor depends on its location in the ventricle: the nutrient vessels are of small caliber and usually originate from the choroidal arteries. ${ }^{27}$

Clinically, pediatric IVMs are usually asymptomatic, until they reach large dimensions in the lateral ventricles, where the risk of hydrocephalus is lower. However, when located in the third or fourth ventricle, obstruction to the flow of cerebrospinal fluid can result in manifestations at early stages of tumor growth. 5,27,29,30 Therefore, symptoms- headache, nausea, vomiting and visual disturbances - are more frequently related to tumor compression and an insidious increase in intracranial pressure., $, 27,31$ Indolent cognitive deficits compromising memory and attention may also occur. ${ }^{32,33}$ Typical symptoms of an acute increase in intracranial pressure are uncommon. ${ }^{29}$ The clinic, therefore, correlates with the location of the tumor within the ventricle, tumor size and the direction of its growth. ${ }^{27}$ Finally, we emphasize that the clinical presentation of the reported patient - convergent strabismus due to paresis of the right lateral rectus muscle - was not correlated with the tumor. This was still relatively small in size and its location did not justify the signs and symptoms.

IVMs usually have the classic radiological appearance of other meningiomas, well-defined globular shape, however no dural tail. They are usually iso to hypotensive on T1-weighted images, hyperintense on T2-weighted images and suffer strong contrast enhancement. ${ }^{16,27}$ Especially in the pediatric population, other more frequent intraventricular tumors can make the differential diagnosis difficult: chorioid plexus tumors, ependymoma, primitive neuroectodermal tumor, teratoma, and astrocytoma. ${ }^{30,34}$ Chorioid plexus tumors usually affect younger than 10 years, and on MRI present a multilobulated mass with intense contrast enhancement and frond-like appearance. Ependymomas represent approximately one third of 
CNS tumors in children under 3 years of age and are characterized by necrosis, hemorrhage, cyst formation, and on $\mathrm{MRI}$, they are hypointense on $\mathrm{T} 1$ and hyperintense and heterogeneous on T2. ${ }^{35}$

The surgical approach of a benign IVM is a neurosurgical challenge, given its deep location and its proximity to eloquent areas and vessels in the ventricular walls.,34 The extent of the initial resection is an independent prognostic factor, presenting a significant association with recurrence and malignancy. ${ }^{2}$ The patient reported here did not present a recurrence in the 4 years of follow-up, which is a result consistent with the literature. In a 2012 review, with 201 cases from different series, there were only eight recurrences; ${ }^{27}$ however, in a meta-analysis with 677 cases of meningiomas in the first two decades, the numbers are more significant: there were 141 recurrences with an average presentation of 3.6 years and with mortality from this event in 46 cases. ${ }^{2}$ The recurrence in this age group occurs basically in cases of atypical meningiomas and anaplastic or after partial resection. ${ }^{36}$ Post-surgical mortality and morbidity in patients in the post-pubertal phase, as is the case of the patient reported here, are similarity to those observed in cases of meningioma in adults. ${ }^{2}$ The use of adjuvant radiotherapy should be avoided in young patients, opting for serial evaluation and reoperation in case of recurrence. . $3,3-8-11,14,18,37^{-37}$

In the literature, there are several surgical approaches for IVM resection: temporoparietal, transfrontal, middle posterior temporal gyrus, posterior inferior temporal gyrus, parieto-occipital and transcallosal., ${ }^{5,34}$ The choice is individualized and based on the location of the tumor within the ventricle, in tumor size and its vascular network, always with a objective of preserving the adjacent brain tissue, performing small corticectomy and retracting little as possible.5,6,27,30,33,34 The justification for the choice the approach is determined by the option that allows better access to the longest axis of the lesion, to minimize transcortical transgression, by the spectrum of preoperative neurological deficits, proximity to the eloquent structures, in addition to anatomical knowledge of the cortical and white matter. ${ }^{29}$ We chose the transcallosal approach because it allows better access to the frontal horn and lateral ventricle body. This approach prevents cortical damage; however, some care is needed with the possible presence of tributary cortical veins tributary of the superior sagittal sinus, which can be anticipated in preoperative examinations, and with the corpus callosum, which must be distinguished from the cingulate gyrus by the change in color. ${ }^{5}$ Despite the degree of difficulty, IVM surgery has shown low morbidity and mortality rates in recent decades, and most postoperative complications- visual deficits and praxis- are temporary. ${ }^{27,29,34}$ These low rates are consistent with the case reported here, which postoperative complications or sequelae did not occur.

\section{Conclusion}

Intraventricular angiomatous meningioma is a rare entity, even more in patients in the first two decades of life. The clinic is nonspecific in most cases, making MRI evaluation necessary for the diagnosis and definition of the surgical approach, and the histopathological analysis is what defines the diagnosis of the angiomatous subgroup. Surgical resection is the treatment of choice. However, the goal of total resection should not be above the goal of preserving the patient's functions and quality of life. 


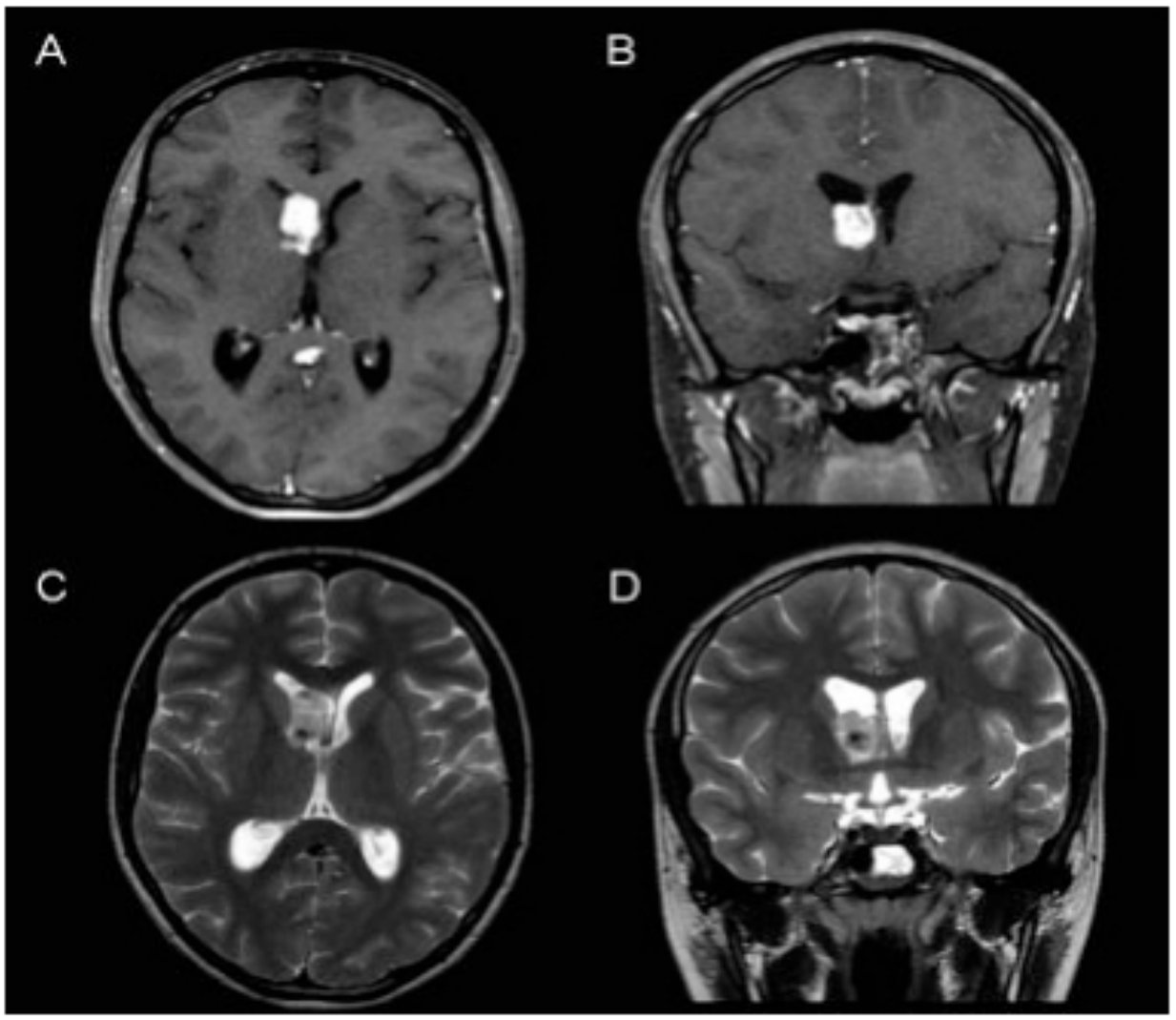

Figure 1: Preoperative MRI: $A$ and $B-T 1$ with gadolinium, axial $(A)$ and coronal $(B)$. Lesion with intense uptake at the level of the right frontal horn, close to the foramen of Monro. $C$ and $D-T 2$ axial(C) e coronal(D). Hyperintense lesion, with a focus of hypointense calcification (also seen in A).

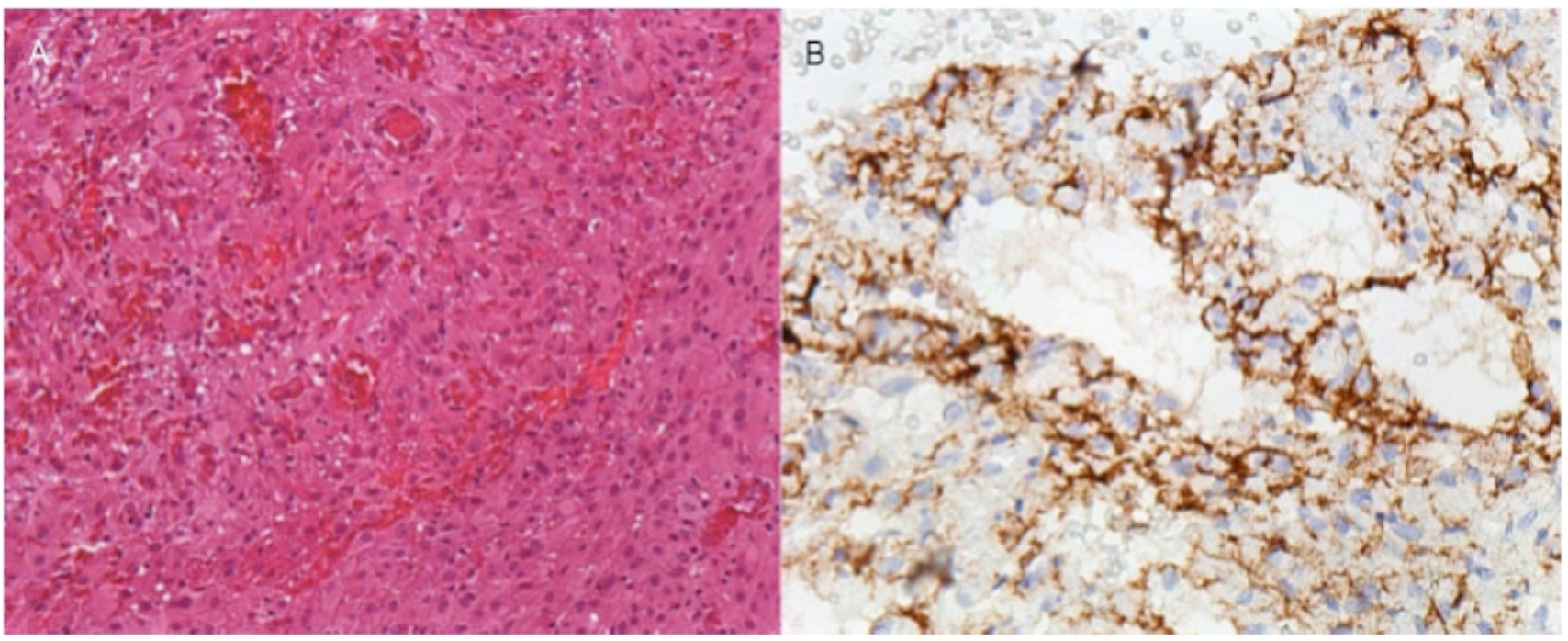

Figure 2: A - Hematoxylin and eosin staining, 200 times magnification: image showing histopathological features of meningioma and significant vascular component. B - Immunohistochemistry, 400-fold magnification: tumor cells showing positivity for EMA. 


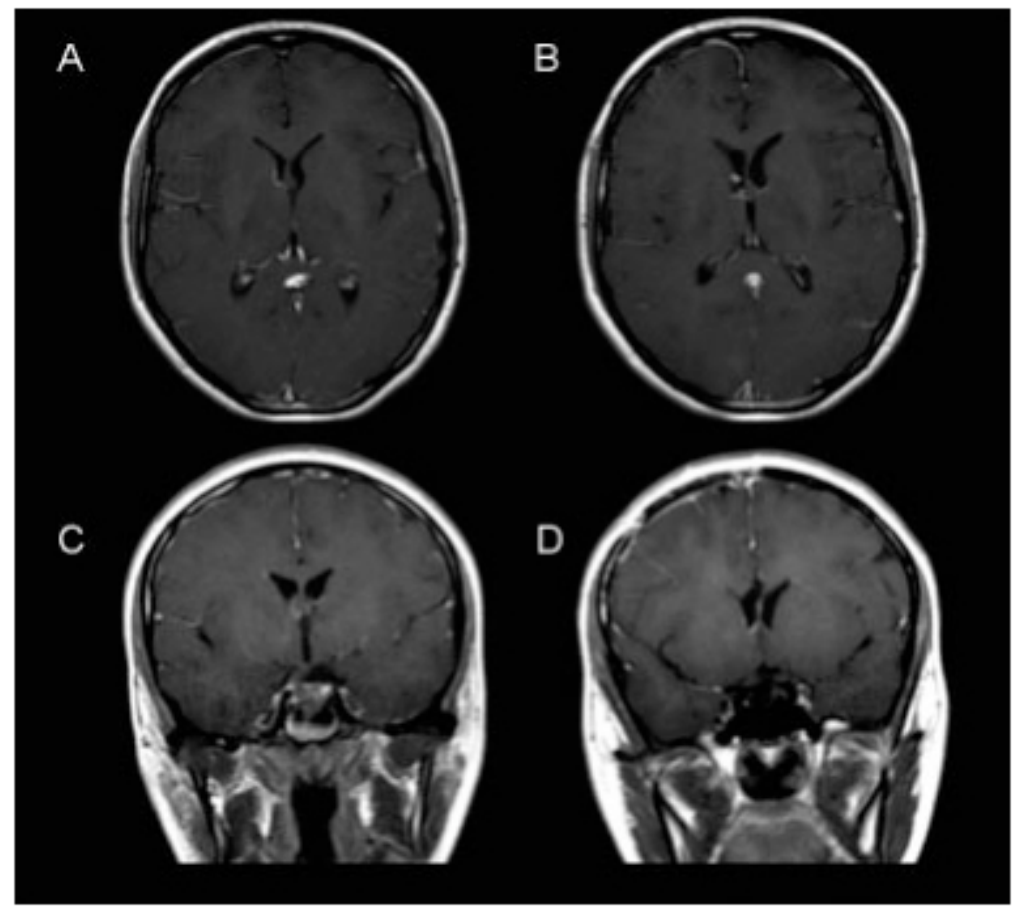

Figure 3: Postoperative MRI: T1 with gadolinium, axial ( $A$ and B) and coronal ( $C$ and $D)$ planes. Small residual focus near the foramen of Monro, attached to the thalamostriate vein (identified during the transoperative period).

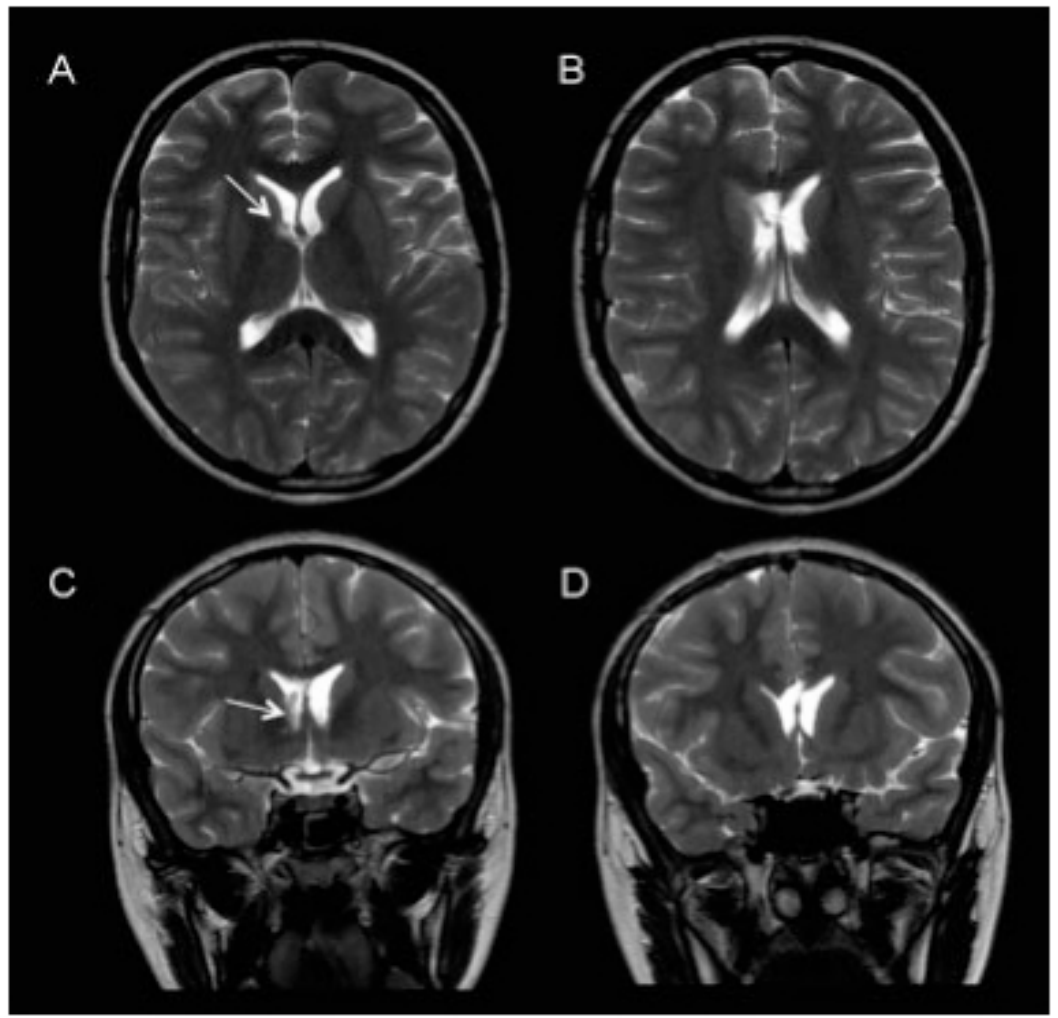

Figure 4: Postoperative MRI: T2 axial ( $A$ and $B)$ and coronal $(C$ and $D)$. Small residual focus at the level of the foramen of Monro (arrows). 


\section{References Références Referencias}

1. Ostrom QT, Gittleman H, Fulop J, et al. CBTRUS Statistical Report: Primary Brain and Central Nervous System Tumors Diagnosed in the United States in 2008-2012. Neuro-oncol 2015;17(Suppl 4): iv1-iv62.

2. Kotecha RS, Pascoe EM, Rushing EJ, et al. Meningiomas in children and adolescents: a metaanalysis of individual patient data. Lancet Oncol 2011;12(13):1229-1239.

3. Menon G, Nair S, Sudhir J, Rao BR, Mathew A, Bahuleyan B. Childhood and adolescent meningiomas: a report of 38 cases and review of literature. Acta Neurochir (Wien) 2009;151(03): 239244, discussion 244.

4. Bhatoe HS, Singh $P$, Dutta V. Intraventricular meningiomas: a clinicopathological study and review. Neurosurg Focus 2006; 20 (03):E9.

5. Nanda A, Bir SC, Maiti T, Konar S. Intraventricular Meningioma: Technical Nuances in Surgical Management. World Neurosurg 2016; 88: 526-537.

6. Lyngdoh BT, Giri PJ, Behari S, Banerji D, Chhabra DK, Jain VK. Intraventricular meningiomas: a surgical challenge. J Clin Neurosci 2007; 14(05): 442-448.

7. Hasselblatt M, Nolte KW, Paulus W. Angiomatous meningioma: a clinico pathologic study of 38 cases.Am J Surg Pathol2004; 28(03): 390-393.

8. Kwee LE, van Veelen-Vincent ML, Michiels EM, Kros $\mathrm{JM}$, Dammers R. The importance of microsurgery in childhood meningioma: a case report. Childs Nerv Syst 2015; 31(01):161- 165.

9. Liu Y, Li F, Zhu S, Liu M, Wu C. Clinical features and treatment of meningiomas in children:report of 12 cases and literature review. Pediatr Neurosurg 2008; 44(02):112-117.

10. Ravindranath K, Vasudevan MC, Pande A, Symss N. Management of pediatric intracranial meningiomas: ananalysis of 31 cases and review of literature. Childs Nerv Syst 2013; 29(04):573-582.

11. Santos MV, Furlanetti L, Valera ET, Brassesco MS, Tone LG, de Oliveira RS. Pediatric meningiomas: a single-center experience with 15 consecutive cases and review of the literature. Childs Nerv Syst 2012;28(11):1887-1896.

12. Cushing HL, Eisenhardt L. Meningiomas: their classification, regional behavior, life history and surgical end results. Charles C Thomas Springfield, III; 1938.

13. Mehta N, Bhagwati S, Parulekar G. Meningiomas in children: A study of 18 cases. J Pediatr Neurosci 2009;4(02):61-65.

14. Arivazhagan A, Devi BI, Kolluri SV, Abraham RG, Sampath S, Chandramouli BA. Pediatricintracrani a I meningiomas-do they differ from their counterparts in adults? Pediatr Neurosurg 2008; 44(01):43-48.
15. Jaiswal S, Vij M, Mehrotra A, Jaiswal AK, Srivastava AK, Behari S. A clinicopathological and neuroradiological study of paediatric meningioma from a single centre. J Clin Neurosci 2011; 18(08): 1084-1089.

16. Whittle IR, Smith C, Navoo P, Collie D. Meningiomas. Lancet 2004; 363(9420):1535-1543.

17. Donne IIMS, Meyer GA, Donegan WL. Estrogenreceptor protein in intracranial meningiomas. J Neurosurg 1979; 50(04):499-502.

18. Kotecha RS, Junckerstorff RC, Lee $\mathrm{S}$, Cole $\mathrm{CH}$, Gottardo NG. Pediatric meningioma: current approaches and future direction. I Neurooncol 2011; 104(01): 1-10.

19. Liu Z,Wang C, WangH,Wang Y,Li JY, LiuY. Clinical characteristics and treatment of angiomatous meningiomas: a report of 27 cases. Int J Clin Exp Pathol 2013;6(04):695-702.

20. Rao S, Rajkumar A, Kuruvilla S. Angiomatous meningioma: a diagnostic dilemma. Indian J Pathol Microbiol 2008;51(01):53-55.

21. Bansal D, Diwaker P, Gogoi P, Nazir W, Tandon A. Intraparenchymal Angiomatous Meningioma: A Diagnostic Dilemma. J Clin Diagn Res 2015;9(10): ED07-ED08.

22. Rathod GB, Vyas K, Shinde P, Goswami SS, Tandan RK. Angioma- tous meningioma in 49 years old male - A rare case report. Int J Curr Microbiol App Sci 2014;3(11):256-260.

23. Meng Y, Chaohu W, Yi L, Jun P, Songtao Q. Preoperative radiologic characters to predict hemangiopericytoma from angiomatous meningioma. Clin Neuro IN eurosurg 2015;138: 78-82.

24. HwangJ, KongD-S, Seol HJ,NamD-H, Lee J-I, ChoiJW. Clinical and Radiological Characteristics of Angiomatous Meningiomas. Brain Tumor Res Treat 2016;4(02):94-99.

25. Ben Nsir A, Chabaane M, Krifa $H$, Jeme $H$, Hattab $\mathrm{N}$. Intracranial angiomatous meningiomas: A 15year, multicenter study. Clin Neurol Neurosurg 2016;149:111-117.

26. Rushing EJ, Olsen $\mathrm{C}$, Mena $\mathrm{H}$, et al. Central nervous system meningiomas in the first two decades of life:a clinicopathological analysis of 87 patients. J Neurosurg 2005;103(6, Suppl)489-495.

27. Ødegaard KM, Helseth E, Meling TR. Intraventricular meningiomas: a consecutive series of 22 patients and literature review. Neurosurg Rev 2013; 36(01): 57-64, discussion 64.

28. Deen HG Jr, Scheithauer BW, Ebersold MJ. Clinical and patholo- gical study of meningiomas of the first two decades of life. J Neurosurg 1982; 56(03): 317-322.

29. Fusco DJ, Spetzler RF. Surgical considerations for intraventricular meningiomas. World Neurosurg 2015; 83(04):460-461. 
30. 30 Liu M, Wei Y, Liu Y, Zhu S, Li X. Intraventricular meninigiomas: a report of 25 cases. Neurosurg Rev 2006; 29(01): 36-40.

31. Rochat $P$, Johannesen $H H$, Gjerris F. Long-term follow up of children with meningiomas in Denmark: 1935 to 1984. J Neurosurg 2004; 100(2, Suppl Pediatrics)179-182.

32. Kashiwazaki D, Takaiwa A, Nagai S, et al. Reversal of cognitive dysfunction by total removal of a large lateral ventricle meningioma: a case report with neuropsychological assessments. Case Rep Neurol 2014; 6(01):44-49.

33. Nakamura M, Roser $F$, Bundschuh O, Vorkapic $P$, Samii M. Intra- ventricular meningiomas: a review of 16 cases with reference to the literature. Surg Neurol 2003; 59(06):491- 503, discussion 503-504.

34. Grujicic D, Cavallo LM, Somma T, et al. Intraventricular Meningiomas: A Series of 42 Patients at a Single Institution and Literature Review. World Neurosurg 2017; 97:178-188 35 Agarwal A, Kanekar S. Intraventricular Tumors. Semin Ultrasound CT MR 2016; 37(02):150-158.

35. Caroli E, Russillo M, Ferrante L. Intracranial meningiomas in children: report of 27 new cases and critical analysis of 440 cases reported in the literature. J Child Neurol 2006;21(01):31-36.

36. Okechi $\mathrm{H}$, Albright AL. Intraventricular meningioma: case report and literature review. Pediatr Neuro surg 2012;48(01):30-34. 